\title{
Caracterización clínica epidemiológica de tuberculosis infantil, Región Sanitaria Metropolitana del Distrito Central, Honduras
}

\author{
Epidemiological and clinical characteristics of tuberculosis in children, Central District Metropolitan Health Region, \\ Honduras.
}

\author{
Ada Yosleny García, ${ }^{1}$ Pedro Luis Jiménez, ${ }^{2}$ Gaspar Rodríguez M. ${ }^{3}$ \\ ${ }^{1}$ Médico General, egresada Facultad de Ciencias Médicas (FCM), Universidad Nacional Autónoma de Honduras (UNAH). \\ ${ }^{2}$ Estudiante Séptimo Año Carrera de Medicina, FCM UNAH. \\ ${ }^{3}$ Médico Especialista en Pediatría; Departamento de Pediatría, Hospital de Especialidades del Instituto Hondureño de Seguridad Social; Tegucigalpa.
}

RESUMEN. Antecedentes: En 2015, la tasa global de incidencia de tuberculosis por 100,000 habitantes fue 43 en Honduras y 29 en el Municipio del Distrito Central. Objetivo: Caracterizar clínica y epidemiológicamente los casos de tuberculosis infantil, Región Sanitaria Metropolitana del Distrito Central (RSMDC), 2016. Métodos: Estudio descriptivo transversal. Se revisaron las Fichas de Notificación de Casos de Tuberculosis en pacientes menor o igual a18 años, registrados durante 2016. Se analizaron variables sociodemográficas y clínicas. Se diseñó una base de datos en Epilnfo 7.2.1. Se obtuvo autorización institucional. La información personal de los casos se manejó confidencialmente. Resultados: Del total de 400 fichas registradas, 13 (3.2\%) contenían información completa. La caracterización se realizó a partir de estos 13 casos, $53.8 \%$ eran niñas, $46.2 \%$ entre 15 y 18 años. En $67 \%$ el diagnóstico fue clínico, $27 \%$ tenía tuberculosis pulmonar y $16.7 \%$ extrapulmonar. El método de diagnóstico más utilizado fue baciloscopía (38.5\%). Todos recibieron tratamiento básico primario, sin comorbilidades, no vivían en condición de riesgo o pertenecían a grupo de riesgo; no presentaron recaídas ni co-infección VIH. Discusión: El sistema de notificación de casos en RSMDC denota un déficit extraordinario e inaceptable en el registro de la información. Aunque la caracterización realizada puede estar sesgada por el número reducido de casos, sus características son similares a lo descrito para la tuberculosis infantil. Es imperativo fortalecer la vigilancia epidemiológica de la tuberculosis en Honduras con énfasis en la tuberculosis infantil y la detección y tratamiento de los contactos.

Palabras clave: Mycobacterium tuberculosis; Tuberculosis; Tuberculosis ganglionar; Tuberculosis pulmonar.

Recibido: 21-11-2018 Aceptado para publicación 20-06-2020

Dirección para correspondencia: Dra. Ada Yosleny García Rodríguez

Correo electrónico: adagarciarodriguez2019@gmail.com

Declaración de relaciones y actividades financieras y no financieras y conflictos de interés: ninguna.

DOI: https://doi.org/10.5377/rmh.v88i1.11596

\section{INTRODUCCIÓN}

La tuberculosis (TB) es una enfermedad infecto-contagiosa y granulomatosa crónica producida por bacterias alcohol-ácido resistentes, que son bacilos aerobios pequeños, de crecimiento lento. ${ }^{1,2}$ Según la Organización Mundial de la Salud (OMS) el porcentaje de niños detectados con TB en el año 2016 fue un millón de casos y 250,000 niños menores de 14 años murieron debido a esta causa, incluidos los niños con TB asociada al VIH. Se calcula que aproximadamente un $6 \%$ de todos los casos nuevos son de Latinoamérica. ${ }^{3,4}$ En Honduras, para el año 2014 la tasa de incidencia de TB fue 43 por 100,000 habitantes y en el municipio del Distrito Central fue 29 por 100,000 habitantes. En 2015, la tasa nacional reportada fue de 32 por 100,000 habitantes. Los tres departamentos con las mayores tasas fueron Gracias a Dios (97.2), Islas de la Bahía (61.1) y San Pedro Sula (44.1). Honduras se encuentra entre los 11 países de la región de las Américas con las tasas más altas de co-infección, $\mathrm{TB}$ y $\mathrm{VIH}{ }^{5}$

La clave para el diagnóstico es buscar sintomáticos respiratorios, detección clínica, antecedentes de convivencia o contacto del niño con un adulto bacilífero, ${ }^{6}$ historia de vacunación de BCG, hallazgos en imágenes radiológicas, tuberculina positiva y aislamiento del bacilo por medio de cultivos ${ }^{7}$ o por biopsia para la TB extrapulmonar si no se puede diagnosticar la causa de la infección mediante métodos más sencillos. La transmisión puede darse a través de la vía transplacentaria, hematógena, aspiración o ingestión de líquido amniótico o secreciones cérvico-vaginales. ${ }^{8}$ Según la edad del niño, las posibles fuentes de contagio son familiares, escuelas, personal de salud, guardería, jardines de infantiles. ${ }^{9}$

La enfermedad en la infancia es más difícil de establecer y controlar por ser inespecífica debido a que el sistema inmune es menos eficiente para contrarrestar el progreso de la infección, así como la diseminación del bacilo a otras partes del cuerpo. ${ }^{10}$ Las formas clínicas se presentan como infección tuberculosa 0 primo infección, forma moderada o común y la forma grave. ${ }^{11}$ Debido a la dificultad que conlleva llegar al diagnóstico en edades tempranas, se han creado métodos como la prueba Xpert 
MTB/RIF, que identifica ADN de Mycobacterium tuberculosis "MTB" y simultáneamente resistencia a rifampicina "RIF", 12 y Xpert Ultra. ${ }^{13}$

En Honduras durante los últimos años, la Secretaría de Salud y socios estratégicos han invertido recursos para mejorar la vigilancia epidemiológica de la TB. El presente estudio se realizó con el objetivo de caracterizar clínica y epidemiológicamente los casos de TB infantil detectados en la Región Sanitaria Metropolitana del Distrito Central (RSMDC), Secretaría de Salud, utilizando la información registrada en la Ficha de Notificación de Casos de TB durante el año 2016.

\section{METODOLOGÍA}

Estudio descriptivo transversal, con un universo conformado por 400 Fichas de Notificación de Casos de TB en pacientes $\leq 18$ años registrados en la RSMDC en el año 2016. Las Fichas de Notificación de Casos fueron registradas en cada uno de los establecimientos de salud al captar sintomáticos respiratorios y enviadas a la RSMDC.

Para la recolección de la información se aplicó un formulario registrando la información sociodemográfica, epidemiológica y clínica registrada en la Ficha de Notificación de Casos incluyendo edad, sexo, procedencia, tipo de TB, métodos diagnósticos, tratamiento, antecedentes de $\mathrm{VIH}$, contacto con personas baciliferas, personas responsables del llenado de la ficha. Se utilizó el programa Epi Info 7.2.1 para diseñar la base de datos. Se analizó la información de forma univariada a través de frecuencias simples y porcentajes.

Para el desarrollo de este estudio, se obtuvo autorización institucional para la revisión de las fichas de notificación de casos. La información personal de los pacientes se manejó con confidencialidad.

\section{RESULTADOS}

Durante el año 2016 se registraron 400 Fichas de Notificación de Casos de TB infantil en la RSMDC. De estas, solamente $3.2 \%$ (13) contaban con información registrada de forma completa. En los cuadros $1 \mathrm{~A}$ y $1 \mathrm{~B}$ se describen las diferentes secciones de la Ficha y la completitud de su llenado. Un $55.2 \%$ (221/400) no tenía el número de registro de identificación de acuerdo con la partida de nacimiento. En un 64\% (256/400) se escribió el antecedente de haber recibido la vacuna de la BCG. En un $100 \%$ de las fichas no se reportó las patologías asociadas, ni co-infección TB/VIH. En el 67\% (268/400) de los casos el método diagnóstico fue clínico, en cuanto a los contactos con el infante, más del $70 \%$ las casillas estaban vacías, se reportó que $93.2 \%$ (373/400) eran pacientes nuevos; a pesar de que son infantes, el esquema de vacunación pediátrico se notificó solo en un $4.5 \%$ (18/400) y el $95.5 \%$ no especificó.

En el Cuadro 2 se describen las características sociodemográficas de los 13 casos con información registrada. Se encontró que, del total el 53.8\% (7/13) correspondían a niñas, $46.1 \%$ (6) pertenecían al grupo de edad de $15-18$ años y $30.7 \%$ (4) al grupo $<5$ años. Los 13 casos procedían de zonas distintas de la
Cuadro 1A. Registro de información en las Fichas de Notificación de Casos de Tuberculosis, Región Sanitaria Metropolitana del Distrito Central, de acuerdo con su completitud, 2016, $n=400$.

\begin{tabular}{|c|c|}
\hline $\begin{array}{l}\text { Items de la ficha de notificación } \\
\text { de casos de tuberculosis }\end{array}$ & $\begin{array}{c}\text { Información registrada } \\
\text { en la ficha } \\
N(\%)\end{array}$ \\
\hline \multicolumn{2}{|l|}{ Datos Generales } \\
\hline Número de registro & $221(55.2)$ \\
\hline Municipio & $345(86.2)$ \\
\hline Código de Unidad de Salud & $366(91.5)$ \\
\hline Número de expediente & 385 (96.2) \\
\hline Región de Salud & $396(99.0)$ \\
\hline Unidad de Salud & $394(98.5)$ \\
\hline CAl & $0(0.0)$ \\
\hline Fecha de notificación & $400(100.0)$ \\
\hline \multicolumn{2}{|l|}{ Identificación del Paciente } \\
\hline Nombre Completo & $400(100.0)$ \\
\hline Sexo & $400(100.0)$ \\
\hline Número de identidad & $221(55.2)$ \\
\hline Embarazo & $0(0.0)$ \\
\hline Fecha de nacimiento & $400(100.0)$ \\
\hline Edad en años cumplidos & $400(100.0)$ \\
\hline Edad en meses & $400(100.0)$ \\
\hline Menor de 5 años, recibió vacuna & $256(64.0)$ \\
\hline BCG & $371(92.7)$ \\
\hline Ocupación & $0(0.0)$ \\
\hline \multicolumn{2}{|l|}{ Tipo de Población } \\
\hline Mestizo & $106(26.5)$ \\
\hline Otros & $0(0.0)$ \\
\hline Dirección Completa (Actual) & $208(52.0)$ \\
\hline Departamento & $400(100.0)$ \\
\hline Municipio & $345(86.2)$ \\
\hline Aldea & $345(86.2)$ \\
\hline Colonia/Barrio & $211(52.7)$ \\
\hline Calle/Avenida & $112(28.0)$ \\
\hline Casa & $112(28.0)$ \\
\hline Otras referencias & $6(1.5)$ \\
\hline Teléfono fijo & $366(91.5)$ \\
\hline Teléfono celular & $109(27.2)$ \\
\hline Cambio de dirección en los últimos 6 meses & $5(1.2)$ \\
\hline Migrante (Interno, Retornado) & $0(0.0)$ \\
\hline Nivel de escolaridad (anotar código) & $307(76.7)$ \\
\hline Paciente trabaja/vive en condición de riesgo & $0(0.0)$ \\
\hline Pertenece el paciente a grupo de riesgo & $0(0.0)$ \\
\hline \multicolumn{2}{|l|}{ Clasificación de Tuberculosis } \\
\hline Pulmonar & $108(27.0)$ \\
\hline Extrapulmonar (sin especificar sitio anatómico) & $69(17.25)$ \\
\hline \multicolumn{2}{|l|}{ Método Diagnóstico } \\
\hline Baciloscopia & $63(15.7)$ \\
\hline Prueba 1 & $63(15.7)$ \\
\hline Prueba 2 & $58(14.5)$ \\
\hline Prueba 3 & $46(11.5)$ \\
\hline Cultivo & $3(0.7)$ \\
\hline Radiografía & $55(13.7)$ \\
\hline Biopsia & $11(2.7)$ \\
\hline Clínico & $268(67.0)$ \\
\hline Otros, Especifique & $0(0.0)$ \\
\hline \multicolumn{2}{|l|}{ Consejería } \\
\hline Recibió consejería & $247(61.7)$ \\
\hline Pre consejería (VIH, TB, TB-MDR) & $247(61.7)$ \\
\hline Post consejería (VIH, TB, TB-MDR) & $203(50.7)$ \\
\hline
\end{tabular}


Cuadro 1B. Registro de información en las Fichas de Notificación de Casos de Tuberculosis, Región Sanitaria Metropolitana del Distrito Central, de acuerdo con su completitud, 2016, $n=400$.

\begin{tabular}{lc}
\hline $\begin{array}{l}\text { Items de la ficha de notificación } \\
\text { de casos de tuberculosis }\end{array}$ & $\begin{array}{c}\text { Información registrada } \\
\text { en la ficha } \\
\mathbf{N}(\%)\end{array}$ \\
\hline Control de Contactos & $11(2.7)$ \\
Convivientes $<15$ años & $28(7.0)$ \\
Convivientes $\geq 15$ años & $10(2.5)$ \\
Habituales $<15$ años & $52(13.0)$ \\
Habituales $\geq 15$ años & $9(2.2)$ \\
Casuales $<15$ años & $21(5.2)$ \\
Casuales $\geq 15$ años & $68(17.0)$ \\
Escolares $<15$ años & $201(50.2)$ \\
Escolares $\geq 15$ años & \\
Condición del Paciente & $373(93.2)$ \\
Nuevo & $0(0.0)$ \\
Recaída, Abandono, Recuperado & $0(0.0)$ \\
Patologías Asociadas ${ }^{\text {a }}$ & \\
Tratamiento & $0(0.0)$ \\
Antecedentes de Tratamiento Previo & \\
Esquema de Tratamiento Actual & $315(93.7)$ \\
Básico Primario & $0(0.0)$ \\
Básico Secundario & $18(4.5)$ \\
Pediátrico & $0(0.0)$ \\
Coinfección TB/VIH & \\
Responsable del llenado de la ficha & $375(93.7)$ \\
Nombre Completo, Cargo & \\
\hline
\end{tabular}

aiabetes Mellitus, Hipertensión Arterial, Insuficiencia Hepática, Insuficiencia Renal, Otros.

Cuadro 2. Características sociodemográficas de niños con tuberculosis, Región Sanitaria Metropolitana del Distrito Central, Honduras, 2016, n= 13.

\begin{tabular}{|c|c|c|c|}
\hline \multirow[b]{2}{*}{ Características } & \multirow[b]{2}{*}{$\begin{array}{l}\text { Total } \\
\mathrm{N}=13 \\
\mathrm{~N}(\%)\end{array}$} & \multicolumn{2}{|c|}{ Sexo } \\
\hline & & $\begin{array}{c}\text { Femenino } \\
\mathrm{N}=7 \\
\mathrm{~N}(\%)\end{array}$ & $\begin{array}{c}\text { Masculino } \\
\mathrm{N}=6 \\
\mathrm{~N}(\%)\end{array}$ \\
\hline \multicolumn{4}{|l|}{ Edad (años) } \\
\hline$<1$ & $1(7.7)$ & $0(0.0)$ & $1(7.7)$ \\
\hline $1-5$ & 3 (23.1) & $1(7.7)$ & $2(15.4)$ \\
\hline $6-10$ & $1(7.7)$ & $0(0.0)$ & $1(7.7)$ \\
\hline $11-14$ & $2(15.4)$ & $2(15.4)$ & $0(0.0)$ \\
\hline $15-18$ & $6(46.0)$ & $4(30.8)$ & $2(15.4)$ \\
\hline \multicolumn{4}{|l|}{ Escolaridad } \\
\hline Primaria completa & $1(7.7)$ & $1(7.7)$ & $0(0.0)$ \\
\hline Primaria incompleta & $2(15.4)$ & $1(7.7)$ & $1(7.7)$ \\
\hline Secundaria completa & $2(15.4)$ & $1(7.7)$ & $1(7.7)$ \\
\hline Secundaria incompleta & $4(30.8)$ & $4(30.8)$ & $0(0.0)$ \\
\hline No aplica & $4(30.8)$ & $1(7.7)$ & $3(23.1)$ \\
\hline \multicolumn{4}{|l|}{ Procedencia } \\
\hline Bernardo Dacy & $1(7.7)$ & $0(0.0)$ & $1(7.7)$ \\
\hline Satélite & $1(7.7)$ & $0(0.0)$ & $1(7.7)$ \\
\hline Nueva Esperanza & $1(7.7)$ & $1(7.7)$ & $0(0.0)$ \\
\hline San Pablo & $1(7.7)$ & $0(0.0)$ & $1(7.7)$ \\
\hline Centro América Oeste & $1(7.7)$ & $1(7.7)$ & $0(0.0)$ \\
\hline La Era & $1(7.7)$ & $1(7.7)$ & $0(0.0)$ \\
\hline Flor del Campo & $1(7.7)$ & $1(7.7)$ & $0(0.0)$ \\
\hline Lomas del Norte & $1(7.7)$ & $1(7.7)$ & $0(0.0)$ \\
\hline Modesto Rodas & $1(7.7)$ & $0(0.0)$ & $1(7.7)$ \\
\hline Reynel Fúnez & $1(7.7)$ & $1(7.7)$ & $0(0.0)$ \\
\hline Villa Centroamericana & $1(7.7)$ & $0(0.0)$ & $1(7.7)$ \\
\hline Zapote Norte & $1(7.7)$ & $1(7.7)$ & $0(0.0)$ \\
\hline Támara & $1(7.7)$ & $0(0.0)$ & $1(7.7)$ \\
\hline
\end{tabular}

Cuadro 3. Características clínicas de niños con tuberculosis, Región Sanitaria Metropolitana del Distrito Central, Honduras, 2016, $n=13$.

\begin{tabular}{lccc}
\hline & Total & \multicolumn{2}{c}{ Sexo } \\
Características & $\begin{array}{c}\mathbf{N}=13 \\
\mathbf{N} \%)\end{array}$ & $\begin{array}{c}\text { Femenino } \\
\mathbf{N}=7\end{array}$ & $\begin{array}{c}\text { Masculino } \\
\mathbf{N}=6\end{array}$ \\
& & $\mathbf{N}(\%)$ & $\mathbf{N}(\%)$ \\
\hline Tipo de TB & & & \\
TB Pulmonar & $7(53.8)$ & $4(30.8)$ & $3(23.1)$ \\
TB Extra pulmonar & $5(38.5)$ & $2(23.1)$ & $3(23.1)$ \\
$\quad$ TB Ganglionar & $1(7.7)$ & $1(7.7)$ & $0(0.0)$ \\
Método Diagnóstico & & & \\
$\quad$ Baciloscopía & $5(38.5)$ & $4(30.7)$ & $1(7.7)$ \\
$\quad$ Radiología & $3(23.1)$ & $1(7.7)$ & $2(15.4)$ \\
$\quad$ PPD & $2(15.4)$ & $1(7.7)$ & $1(7.7)$ \\
$\quad$ Cultivo & $2(15.4)$ & $1(7.7)$ & $1(7.7)$ \\
$\quad$ Biopsia & $1(7.7)$ & $0(0.0)$ & $1(7.7)$ \\
Contacto con & & & \\
pacientes baciliferos & & & \\
Si & $0(0.0)$ & $0(0.0)$ & $0(0.0)$ \\
$\quad$ No & $13(100)$ & $7(100.0)$ & $6(100.0)$ \\
VIH & & & \\
Si & $0(0)$ & $0(0.0)$ & $0(0.0)$ \\
No & $13(100)$ & $7(100.0)$ & $6(100.0)$ \\
\hline
\end{tabular}

RSMDC. Al caracterizar clínicamente a los infantes estudiados, se identificó que $53.8 \%$ (7) de los casos presentó TB pulmonar y $46.1 \%$ (6) casos de TB extra pulmonar. (Ver Cuadro 3).

Los métodos diagnósticos utilizados fueron en un 38.5\% (5) Baciloscopía, 23.1\% (3) Radiografía, 15.4\% (2) PPD y Cultivo (no especificaron lugar de toma del cultivo) y $7.4 \%$ Biopsia. El 23.0\% (3) de los infantes convivía en sus casas con 7 personas, en hacinamiento, sin antecedentes de TB. No se reportó presencia de recaídas en el periodo de estudio, no tenían antecedentes de co-infección TB/VIH (Ver Cuadro 3). Se informó que un establecimiento de cuarto nivel, el Instituto Cardio Pulmonar diagnosticó un 53.8\% (7) de los infantes y el Instituto Hondureño de Seguridad Social diagnosticó 23.0\% (3). De los establecimientos de segundo nivel: El Carrizal, El Tizatillo y Nueva Esperanza diagnosticaron 7.6\% (1) cada uno.

Todos pacientes reportados en las fichas recibieron tratamiento básico primario, no tenían comorbilidades ni vivían en alguna condición de riesgo o pertenecían a un grupo de riesgo. Los datos de los pacientes fueron registrados por una enfermera profesional en $69.2 \%$ (9) de los niños, $15.3 \%$ (2) por auxiliares de enfermería y 2 no estaban consignados. Recibieron pre consejería sobre TB, VIH y TB Multi drogo resistente en un $92.3 \%$ (12) de los casos y post consejería $61.5 \%$ (8).

\section{DISCUSIÓN}

Los niños representan aproximadamente el $10 \%$ de los casos de TB en todo el mundo, y los menores de cinco años son particularmente susceptibles a las formas graves de enfermedad, ${ }^{14,15}$ En México una investigación describe que la edad de presentación de TB infantil muestra un comportamiento bimodal: una mayor incidencia en los menores de dos años, una disminución de la incidencia entre los 5 y 10 años, y un incremento 
nuevamente en la adolescencia, ${ }^{16}$ lo cual coincide con nuestro estudio al mostrar mayor incidencia entre 15-18 años, menor incidencia entre 5-10 años y difiere con los menores de 2 años ya que muestra menor incidencia.

En el presente estudio se muestra que el sexo femenino es más frecuente en el rango de edad de $15-18$ años con $30.8 \%$ (4/13) casos de TB a diferencia de un estudio realizado en Cuba en 2012, que reportó el predominio del sexo masculino con un $59 \%$ (19) casos, en el mismo rango de edad, ${ }^{17}$ y un estudio en Brasil en 2019 en el cual fue posible observar que el $56 \%$ de los niños y adolescentes eran del sexo masculino. ${ }^{18}$

En el estudio de Brasil antes mencionado el tipo más común fue TB pulmonar en un $59 \%$, seguido de TB extra pulmonar $25 \%$ y mixto en un $13 \% .{ }^{18}$ Otro estudio en España muestra que el $90 \%$ presenta afectación pulmonar, ${ }^{19}$ estudios con los cuales concuerdan con los presentes hallazgos ya que este estudio reportó 53.8\% (7) casos de TB pulmonar y $46.1 \%$ (6) casos de TB extra pulmonar y dentro de esta clasificación 1 caso de TB ganglionar.

Este estudio muestra que el método diagnóstico frecuente utilizado fue la Baciloscopía en $38.5 \%$ (5), seguido de la radiografía $23 \%$ (3), estos datos concuerdan con un estudio en Chile que afirma que la baciloscopía continúa siendo internacionalmente la herramienta primaria en el diagnóstico de la TB pulmonar activa; esta es la prueba más utilizada no sólo en la búsqueda de casos infecciosos de la comunidad, sino además como medidor de la eficacia del tratamiento en estos pacientes,$^{20}$ la radiografía es la modalidad de imagen utilizada para identificar pacientes con estado pulmonar anormal, según un estudio en Brasil; este tipo de imagen ha sido útil para evaluar lesiones pulmonares incluso en pacientes asintomáticos. ${ }^{21} \mathrm{En}$ Honduras actualmente, la prueba de tuberculina es indicada e interpretada por profesionales competentes para tratar de evitar los diagnósticos falsos negativos y ante la duda se debe repetir la prueba de 4 a 6 semanas después, en las fichas analizadas para el estudio no se detalla su utilización.

En Chile 2016 mostraron un estudio que el 33,3\% de los casos tiene como factor de riesgo contactos baciliferos positivos, ${ }^{22}$ en este estudio no se constató algún paciente en contacto con personas con TB activa 0 antecedentes de la misma; el apartado de contactos registrados en la ficha de notificación solo permite conocer el sexo, la edad y el tipo de contacto (conviviente, habitual, casual, laboral, escolar), sin especificar si estos contactos eran TB positivos.

En Sao Paulo para el año 2015, hubo 6,800 personas diagnosticadas con VIH y TB, de ellas, más de 1,000 personas desarrollaron TB multi-rresistente (TB-MDR) 0 ampliamente TB resistente a los medicamentos (XDR-TB). Nuestro estudio no reportó ningún caso. ${ }^{23}$

En 2015 el número mundial estimado de nuevos casos (incidentes) de TB fue 10,4 millones, de los cuales 1,0 millón (10\%) eran niños. ${ }^{24}$ En 2014 en Europa desarrollaron la enfermedad 219.000 niños, siendo España el país con mayor número de casos pediátricos (303 casos), con una incidencia en 2014 de $4.3 / 100,000$ por habitantes.$^{25} \mathrm{~A}$ diferencia de otros países, Honduras no posee datos estadísticos publicados de TB infantil.

La población infantil es un grupo especialmente vulnerable con mayor riesgo de progresión a enfermedad activa tras la infección, mayor riesgo de desarrollar formas graves, además de que el diagnóstico es más difícil que en adultos por lo que se torna critico tener un sistema de vigilancia robusto que permita la toma de decisiones, la aplicación efectiva y oportuna de medidas para alcanzar la meta de cero muertes en niños por TB.

El sistema de notificación de casos en RSMDC denota un déficit extraordinario e inaceptable en el registro de la información, pudo observarse que, de los 400 casos de infantes reportados en las fichas de notificación nacionales, solo 13 contenían datos completos, lo que contribuye a invisibilizar la magnitud real de la epidemia de TB infantil, la que puede ser más grave de lo que se estima actualmente.

En conclusión, aunque la caracterización realizada puede estar sesgada por el número reducido de casos, sus características son similares a lo descrito para la TB infantil. Es imperativo fortalecer la vigilancia epidemiológica de la TB en Honduras con énfasis en la TB infantil específicamente en la detección, notificación, diagnóstico de los niños y el tratamiento de los contactos. Por tanto, recomendamos a las autoridades fortalecer la búsqueda y registro adecuado de los casos y divulgar la situación epidemiológica con un plan de mejora que pueda ser supervisado y monitoreado.

\section{CONTRIBUCIONES}

AYG y GRM concibieron el estudio. GRM diseñó el estudio. PLJ lideró la recolección y análisis de la información. AYG lideró la redacción del artículo. Todos los autores atendieron las recomendaciones editoriales, revisaron y aprobaron la versión final del artículo.

\section{AGRADECIMIENTOS}

Se agradece a la Dra. Alejandra García, Médico General, egresada de la Universidad Católica de Honduras (UNICAH), por su colaboración en la recopilación de información de fichas de notificación.

\section{REFERENCIAS}

1. Organización Mundial de la Salud. Tuberculosis [Internet]. Ginebra: OMS; 2018 [consultado 10 octubre 2019]. Disponible en: http://www.who.int/topics/tuberculosis/es/

2. Secretaria de Salud (HN). Manual de normas de control de la tuberculosis. [Internet]. Tegucigalpa: Secretaría de Salud; 2012 [consultado 10 octubre 2019]. Disponible en: http://www.bvs.hn/Honduras/Postgrados/NormasTBMarzo2013.pdf

3. Tovar Díaz M, Tang Velásquez AM, Concha Mendoza ND. Tuberculosis extrapulmonar en pediatría: un reto diagnóstico. Med UIS [Internet]. 2013 [consultado 10 octubre 2019];26(1):45-58.Disponible en: https://revistas. uis.edu.co/index.php/revistamedicasuis/article/view/358

4. Centro Nacional de Información de Ciencias Médicas (CU), Biblioteca Médica Nacional. Honduras: indicadores de Salud. Fotográfico de Salud [Internet]. 2017[consultado 10 octubre 2019];3(1):1-12. Disponible en: http://files.sld.cu/bmn/files/2016/12/factografico-de-salud-enero-2017.pdf

5. Organización Panamericana de la Salud. Estrategia de cooperación de 
país de la OPS-OMS: Honduras, 2017-2021 [Internet]. Ginebra: OPS; 2017 [consultado 21 mayo 2019]. Disponible en: https://www.paho.org/es/ documentos/estrategia-cooperacion-pais-opsoms-honduras-2017-2021

6. Méndez Echevarria A, Mellado Peña MJ, Baquero Artigao F, García Miguel MJ. Tuberculosis. En: Protocolos diagnóstico terapéuticos de Infectología Pediátrica SEIP-AEP. [Internet]. Madrid: Ergon; 2011. p.103-112. [consultado 21 mayo 2019]. Disponible en: https://goo.gl/ZU97TP

7. Bisero E, Luque G, Borda ME, Melillo K, Zapata A, Varela S. Tuberculosis en una población pediátrica atendida en un hospital público. Adherencia al tratamiento. Estudio descriptivo. Rev Am Med Resp [Internet]. 2013[consultado 21 mayo 2019];13(4):184-9. Disponible en: http://www.redalyc.org/ articulo.oa?id=382133980003

8. Muñoz Peña R, Reynel PGE, Abreu Suárez G, González Valdés JA, Rodríguez vargas LE. Control de foco de tuberculosis en menores de 15 años. Rev Cubana Pediatr. 2013;86(2):189-199

9. Varela-Martínez C, Yadon ZE, Marín D, Heldal E. Contrasting trends of tuberculosis in the cities of San Pedro Sula and Tegucigalpa, Honduras, 2005-2014. Rev Panam Salud Publica. 2016;39(1):51-9.

10. Organización Mundial de la Salud. Tuberculosis Infantil [Internet]. Ginebra: OMS; 2018 [consultado 21 mayo 2019]. Disponible en: http://www.who.int/ tb/challenges/children/es/

11. Sociedad Argentina de Pediatría. Criterios de diagnósticos y tratamiento de la tuberculosis infantil [Internet]. Buenos aires: SAP; 2014 [consultado 21 mayo 2019]. Disponible en: http://www.sap.org.ar/uploads/consensos/ criterios-de-diagn-oacutestico-y-tratamiento-de-la-tuberculosis-infantilnbsp2016.pdf.

12. Ghussn Cano AP, Neves Romaneli MT, Mendes Pereira R, Teresinha AT. Tuberculose em pacientes pediátricos: como tem sido feito o diagnóstico?. Rev Paul Pediatr [Internet]. 2017 [consultado 21 mayo 2019];35(2): 165-70. Disponible en: https://www.scielo.br/scielo.php?pid=S0103$05822017000200165 \&$ script $=$ sci abstract\&tlng $=$ pt

13. Delgado ML, González NE. Comparación de la capacidad predictiva de dos escalas para el diagnóstico de tuberculosis en niños. Arch Argent Pediatr. 2015;113(6):491-7.

14. Schmidt CM, Lovero KL, Carvalho FR, dos Santos CMD, Barros AC, Quintanilla AP, et al. Serum anti-Mce1A immunoglobulin detection as a tool for differential diagnosis of tuberculosis and latent tuberculosis infection in children and adolescents. Tuberculosis [Internet] 2020 [consultado 4 abril 2019];120;101893. Disponible en: https://doi.org/10.1016/j. tube.2019.101893

15. Daley CL. The Global Fight Against Tuberculosis. Thorac Surg Clin [Internet];2019 [consultado 14 julio 2019];29(1);19-25. Disponible en: https:/l www.thoracic.theclinics.com/article/S1547-4127(18)30137-3/fulltext

16. Macías Parra M. Tuberculosis pediátrica. Bol Med Hosp Infant Mex [Internet]. 2017 [consultado 14 julio 2019];74(1):1-2. Disponible en: https://doi. org/10.1016/j.bmhimx.2017.01.003

17. Coro González R, Abreu Suárez G, Muñoz Peña R, González Valdés JA, Rodríguez Vargas LE. Comportamiento de la tuberculosis en adolescentes de 15 a 18 años. Rev Cubana Pediátr [Internet]. 2012 [consultado 14 julio 2019];84(3):225-233. Disponible en: http://scielo.sld.cu/scielo. php?script=sci arttext\&pid=S0034-75312012000300003\&lng=es.

18. Bezerra Sousa GJ, de Oliveira Silva JC, de Queiroz TV, Guedes Bravo L, Branco Brito GC, de Souza Pereira A, et al. Clinical and epidemiological features of tuberculosis in children and adolescents. Rev Bras Enferm [Internet]. 2019 [consultado 14 julio 2019];72(5):1271-8. Disponible en: https://doi.org/10.1590/0034-7167-2018-0172.

19. Rivero Calle I. Tuberculosis en la edad pediátrica. [Internet]. Madrid: AEPap; 2019. [consultado 14 julio 2019]. Disponible en: https://www. aepap.org/sites/default/files/documento/archivos-adjuntos/introduccion tuberculosis_21marzo.pdf

20. Sardiñas M, García G, Martínez MR, Díaz R, Mederos LM. Importancia del control de la calidad de la baciloscopia en los laboratorios de diagnóstico de tuberculosis. Rev Chil Infectol [Internet]. 2016 [consultado 21 mayo 2019];33(3):282-6. Disponible en: https://scielo.conicyt.cl/scielo. php?script=sci arttext\&pid=S0716-10182016000300005

21. de Oliveira M, Duarte SB, Giacomini G, Marques Pereira PC, de Souza LR, Arruda Miranda JR, et al. A lung image reconstruction from computed radiography images as a tool to tuberculosis treatment control. J Venom Anim Toxins Incl Trop Dis [Internet]. 2019 [consultado 14 julio 2019];25:e144918. Disponible en: https://www.scielo.br/scielo.php?script=sci_arttext\&pid $=$ S1678-91992019000100302

22. Ministerio de Salud (Cl). Tuberculosis informe de situación : Chile: 2016. Santiago: Ministerio de Salud; 2017.

23. Calçada Carvalho AC, Araújo Cardoso CA, Martire TM, Migliori GB, Sant'Anna CC. Epidemiological aspects, clinical manifestations, and prevention of pediatric tuberculosis from the perspective of the End TB strategy. J Bras Pneumol [Internet]. 2018 [consultado 14 julio 2019];44(2):134144. Disponible en: https://doi.org/10.1590/s1806-37562017000000461

24. Organización Mundial de la Salud. Informe Mundial sobre la Tuberculosis 2016: sinopsis. [Internet]. Ginebra: OMS; 2016 [consultado 14 julio 2019]. Disponible en: https://www.who.int/tb/publications/global_report/gtbr2016 executive summary es.pdf

25. Mellado Peña MJ, Santiago García B, Baquero-Artigao F, Moreno Pérez D, Piñeiro Pérez R, Méndez Echevarría A, et al. Actualización del tratamiento de la tuberculosis en niños. An Pediatr. 2018;88(1):52.e1-52.e12.

ABSTRACT. Background: In 2015, the overall tuberculosis incidence rate per 100,000 inhabitants was 43 in Honduras and 29 in the Central District Municipality. Objective: To characterize clinically and epidemiologically the cases of childhood tuberculosis, Metropolitan Sanitary Region of the Central District (RSMDC), 2016. Methods: Descriptive cross-sectional study. We reviewed the Tuberculosis Case Notification Forms for patients under or equal to 18 years of age, registered during 2016. Sociodemographic and clinical variables were analyzed. A database was designed in Epilnfo 7.2.1. Personal case information was handled confidentially. Results: Of the total 400 registered cases, $13(3.2 \%)$ contained complete information. Based on these 13 cases, $53.8 \%$ were girls, $46.2 \%$ between 15 and 18 years old. The diagnosis was clinical in $67 \%$, $27 \%$ had pulmonary tuberculosis and $16.7 \%$ extrapulmonary. The most commonly used diagnostic method was baciloscopy (38.5\%). All received basic primary treatment, without comorbidities, were not at risk or belonged to a risk group; they did not present relapses or HIV co-infection. Discussion: The case notification system in RSMDC denotes an extraordinary and unacceptable deficit in the recording of information. Although the characterization made may be biased by the small number of cases, its characteristics are similar to those described for childhood tuberculosis. It is imperative to strengthen epidemiological surveillance of tuberculosis in Honduras with emphasis on child tuberculosis and contact detection and treatment.

Keywords: Lymph node; Mycobacterium tuberculosis; Tuberculosis; Tuberculosis pulmonary. 